\title{
Low side lobe tapered slot antenna with high gain using gradient refractive index metamaterial for ultra wideband application
}

\author{
R. Singha ${ }^{1}$, D. Vakula ${ }^{2}$ \\ Electronics and Communication Engineering, National Institute of Technology, Warangal 506004, India \\ *corresponding author, E-mail: ieee.rahul5488@gmail.com
}

\begin{abstract}
A novel broadband gradient refractive index (GRIN) metamaterial is used to improve the gain of the tapered slot antenna. The proposed metamaterial is capable of reducing the side lobe level of the antenna. The gradient refractive index (GRIN) metamaterial is constructed by using nonresonant parallel-line unit cells with different refractive index. Due to the non-resonant structure, the proposed unit cell exhibits low loss and large frequency bandwidth. The metamaterial, effective refractive index is lower than that of the substrate on which the antenna is printed. The GRIN metamaterial is integrated in front of the antenna which has the capability to manipulate electromagnetic wave accurately. The measurement results indicate that the reflection coefficient of the antenna is below $-10 \mathrm{~dB}$ over the frequency band from 3 to $11 \mathrm{GHz}$. The radiation pattern of the antenna shows the beam width becomes narrow and directive with low side lobe level. The peak gain is increased by $2.1 \mathrm{~dB}$ at $9.5 \mathrm{GHz}$. The antenna has shown significant improvement on gain at low frequency.
\end{abstract}

\section{Introduction}

The traditional Vivaldi antenna is a kind of traveling wave antenna with non-resonant characteristics. Therefore, Vivaldi antenna can provide large bandwidth. Vivaldi planar antenna has light weight, low cost, ease of fabrication and capable of integration with other electronics systems. This antenna is also called as tapered slot antenna (TSA) [1]. TSAs provide directional end fire radiation patterns with simple structures. TSAs have wide application in military and medical fields, such as microwave imaging, ground penetrating radar, tumor detection etc. In printed TSA, the phase velocity of the traveling wave is less than the velocity of light. Therefore, the directivity of the antenna is limited. So, large size is required to get high gain.

To eliminate these problems, low refractive index metamaterial is widely used as a beam focusing lens. The low or zero index metamaterial (LIM/ZIM) is a good option for all broadband antennas. The lens can help to improve the radiation pattern and gain of any antenna over a broader frequency range. Several works are published in the literature based on these techniques applied to Vivaldi antennas. In [2], the directivity of the traditional Vivaldi antenna is increased by using anisotropic zero-index metamaterials (ZIM). By using resonant meander-line structure an anisotropic ZIM is designed over working frequency range $9.5-10.5 \mathrm{GHz}$, which is integrated with the Vivaldi antenna smoothly. The gain of the antenna is increased approximately to $3.6 \mathrm{~dB}$. But the size of the antenna is very large and ZIM is used for a narrow band. A broadband artificial material is designed by using nonresonant parallel-line unit cell [3]. The proposed material is integrated with an antipodal tapered slot antenna (ATSA). The gain of the ATSA is increased, but the side lobes of the antenna pattern are also increased. To reduce the side lobe, an exponential slot edge with kotch fractal antipodal Vivaldi antenna (ESE-AVA) are designed [10], [11]. But the reflection coefficient of the antennas are fluctuating and goes above $-10 \mathrm{~dB}$ at some frequency, indicting a poor impedance matching. Moreover, the gain is also low varying at $8 \mathrm{~dB}$ over the operating frequency band.

In the proposed work, a broadband gradient refractive index (GRIN) lens is designed by using broadband nonresonant parallel-line metamaterial. The metamaterial is introduced in the extended substrate of TSA which is not attempted in published literature. The designed GRIN lens will work over 2-12 GHz. The measurement results of the GRIN loaded TSA shown improvement in gain at high as well as low frequencies.

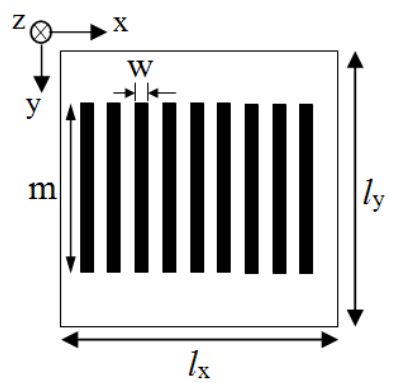

(a)

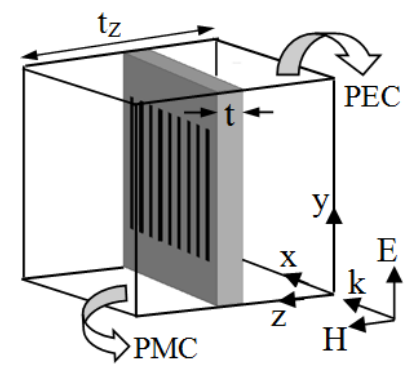

(b)
Figure 1: The geometry (a) unit cell metamaterial, (b) its simulation model. The dimensions of unit cell are $l_{x}=l_{y}=6$ $\mathrm{mm}, w=0.2 \mathrm{~mm}, t=0.8 \mathrm{~mm}$ and $t_{z}=6 \mathrm{~mm}$.

\section{Design and Characteristics of GRIN Metamaterial}

To construct GRIN, it is necessary to implement and verify the unit cell metamaterial. Here, the unit cell is designed by using parallel strip lines on the substrate as shown in Fig. 1(a). The proposed material is printed on the Roger RO4003 
(dielectric constant 3.55) with a substrate thickness of 0.8 $\mathrm{mm}$ and loss tangent 0.0027 . To obtain an array of parallelline unit cells, it is essential to define periodic boundary conditions. The perfect electric conductor (PEC) and perfect magnetic conductor (PMC) boundary conditions are defined in Fig. 1(b). The electric and magnetic polarizations are along the $y$ and $z$-axes, respectively. EM wave propagation is considered along $\mathrm{x}$-axis. By applying the effective medium theory, the effective parameters are extracted from the scattering parameters. The proposed metamaterial parameters such as effective refractive index $(n)$, permittivity $\left(\varepsilon_{y}\right)$ and permeability $\left(\mu_{z}\right)$ are obtained via retrieval formula described in [5].

Fig. 2 shows the magnitude and phase of the scattering parameters for the parallel-line unit cell metamaterial for $m=4 \mathrm{~mm}$ and $1.1 \mathrm{~mm}$, where $m$ is length of unit cell. The electrical length of the parallel metallic line is different for various length of $m$. Therefore, the resonant frequency of the parallel-line metamaterial values are also change. The resonant frequencies are obtained as $14 \mathrm{GHz}$ and $22 \mathrm{GHz}$ for $m=4 \mathrm{~mm}$ and $1.1 \mathrm{~mm}$, respectively.

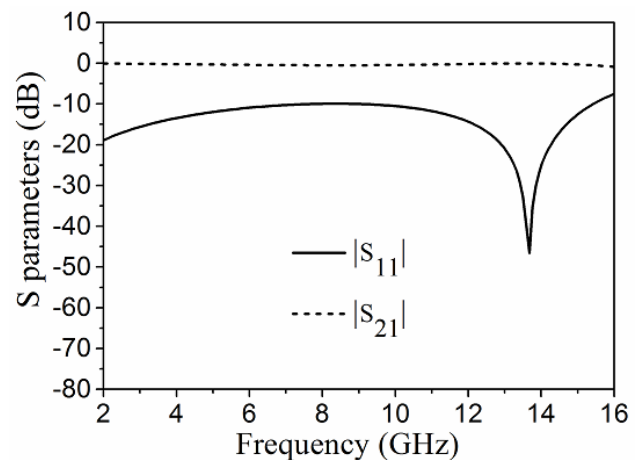

(a)

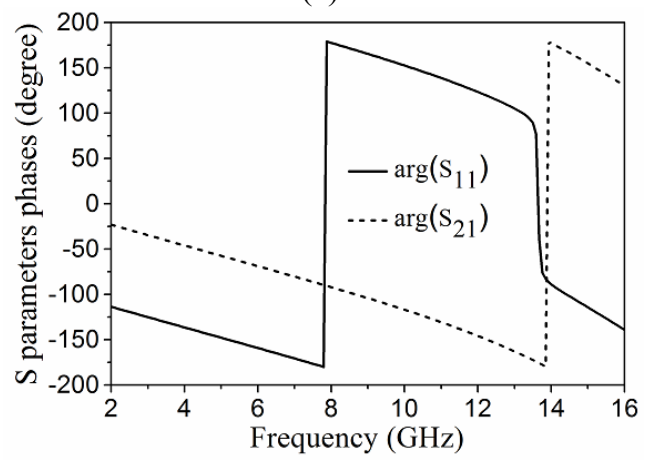

(b)

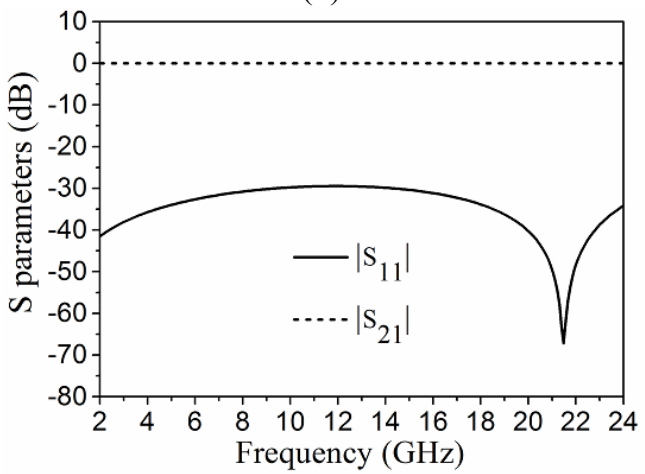

(c)

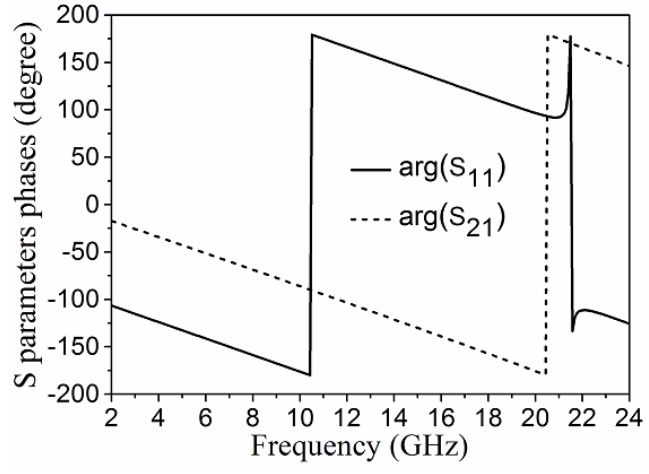

(d)

Figure 2: Scattering parameters response of the unit cell metamaterial, (a) Magnitude response for $m=4 \mathrm{~mm}$, (b) phase response for $m=4 \mathrm{~mm}$, (c) Magnitude response for $m=1.1 \mathrm{~mm}$, and (d) phase response for $m=1.1 \mathrm{~mm}$.

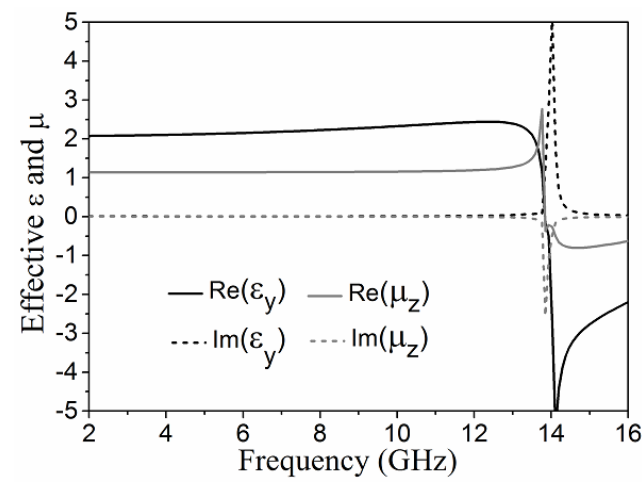

(a)

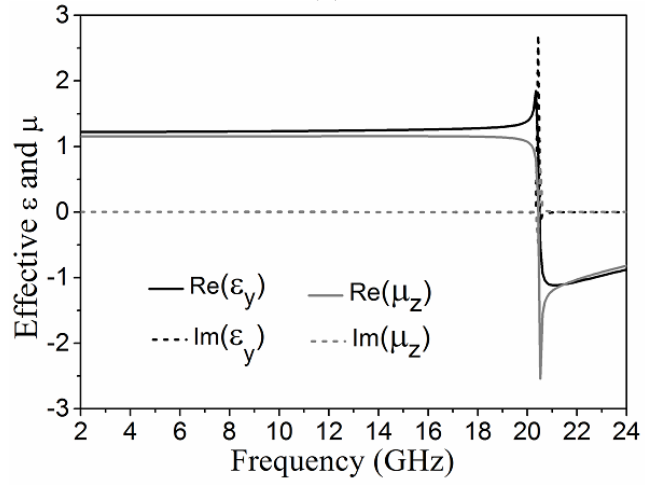

(b)

Figure 3: Effective parameters of the unit cell metamaterial, (a) Effective permittivity $\left(\varepsilon_{y}\right)$ and permeability $\left(\mu_{z}\right)$ for $m=4$ $\mathrm{mm}$ and (b) Effective permittivity $\left(\varepsilon_{y}\right)$ and permeability $\left(\mu_{z}\right)$ for $m=1.1 \mathrm{~mm}$.

The corresponding extracted permittivity and permeability results are illustrated in Fig. 3 . The effective permittivity is marked $2+0.013 \mathrm{i}$ at $3 \mathrm{GHz}$ and $2.3+0.011 \mathrm{i}$ at $10 \mathrm{GHz}$, as shown in Fig. 3(a). Similarly, the imaginary parts of the effective parameters are nearly zero, as illustrated in Fig. 3(b). It is clearly shows that the proposed parallel-line unit cell has low losses over a non-resonant broadband region. 


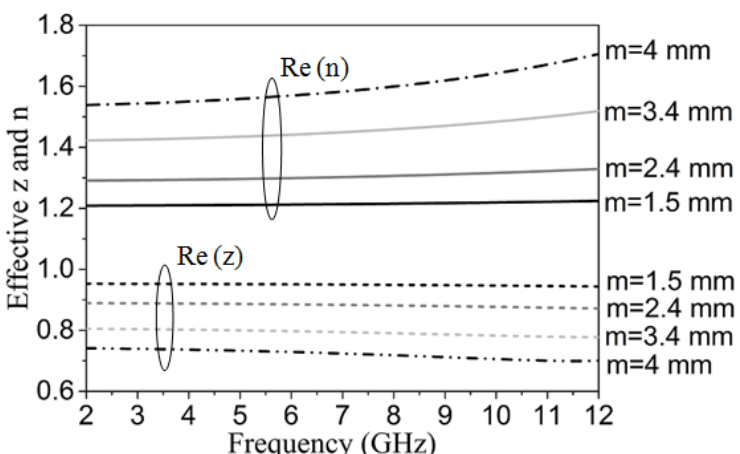

(a)

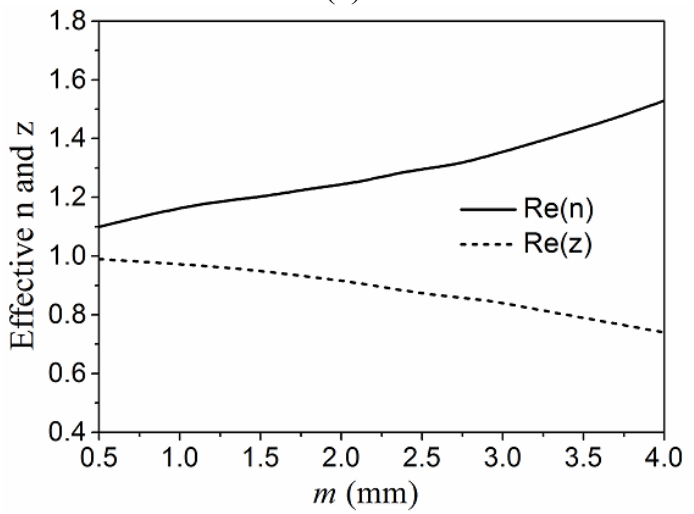

(b)

Figure 4: Retrieval results for the parallel line medium, (a) The extracted index and impedance for several values of $m$, (b) The relationship between the extracted refractive index $(n)$ and wave impedance $(z)$ on length $m$.

To understand the effect of length on parallel-line metamaterial, the extracted refractive index and impedance for four different unit cells with the lengths of $m=4 \mathrm{~mm}$, $3.4 \mathrm{~mm}, 2.4 \mathrm{~mm}$ and $1.5 \mathrm{~mm}$ are shown in Fig. 4(a). In Fig. 4(b), it is observed that the effective refractive index value becomes low by decreasing the length of the parallel metallic line $(m)$. Moreover, the effective impedance values are near to the unity when the value of $m$ is reduced.

\section{Design of GRIN TSA}

Fig. 5 shows the geometry of the reference tapered slot antenna with dimensions. The proposed antenna is printed on Roger RO4003 substrate with dielectric constant 3.55, tangent loss 0.0027 and a substrate thickness of $0.8 \mathrm{~mm}$. The antenna substrate is extended by $60 \mathrm{~mm}$ for parallel-line metamaterial. The excitation of the antenna is given by using microstrip line with impedance transition techniques. The width of microstrip line transition varies from $1.7 \mathrm{~mm}$ to $0.8 \mathrm{~mm}$. The exponential inner curves adopted in this design are described by the equation

$y= \pm(0.0203096832 * \exp (0.15 \mathrm{x})+0.07044958531)$

where, $0.1 \mathrm{~mm} \leq \mathrm{y} \leq 15 \mathrm{~mm}$ and $2.5 \mathrm{~mm} \leq \mathrm{x} \leq 41.5 \mathrm{~mm}$.

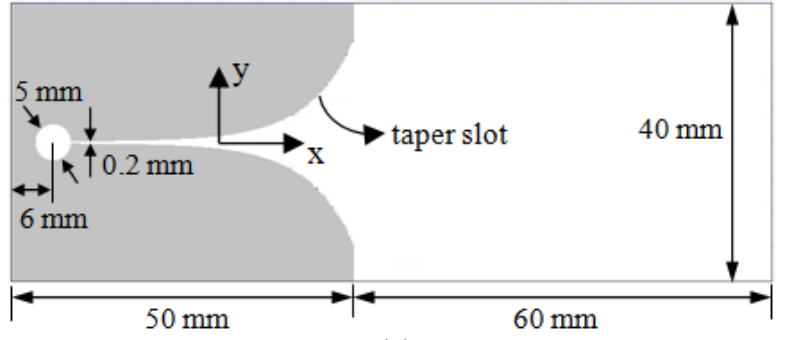

(a)

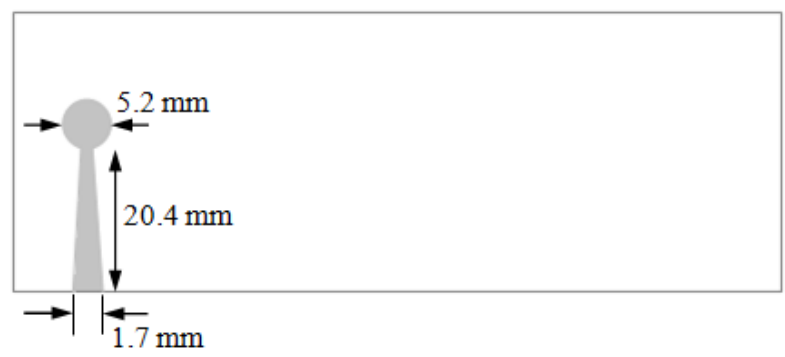

(b)

Figure 5: Geometry of the reference TSA, (a) Top view and, (b) Bottom view

TSA starts to radiate when its output aperture width is half-wavelength [6]. To improve impedance matching of the antenna, microstrip-line includes a small matching section. Hence, the reference TSA structure consists of a feed line, a microstrip-slot line transition and a radiating slot. Microstrip-slot line transition exhibits a frequencyindependent transition characteristic of electromagnetic fields from the microstrip line to the slotline and vice versa [7].

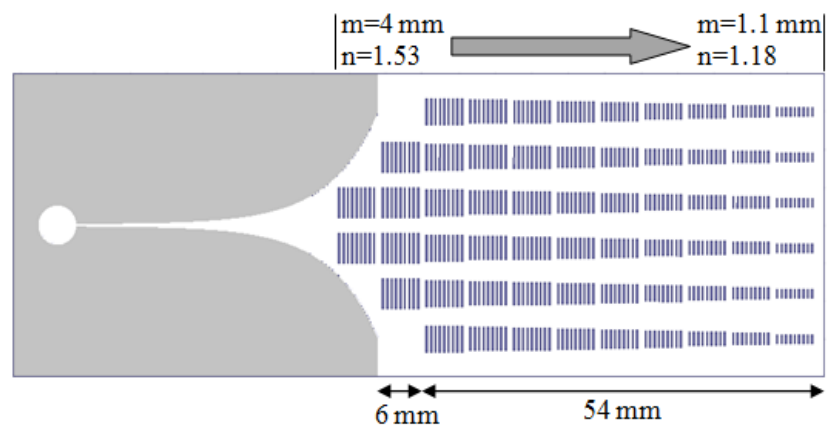

Figure 6: Metamaterial GRIN integrated TSA

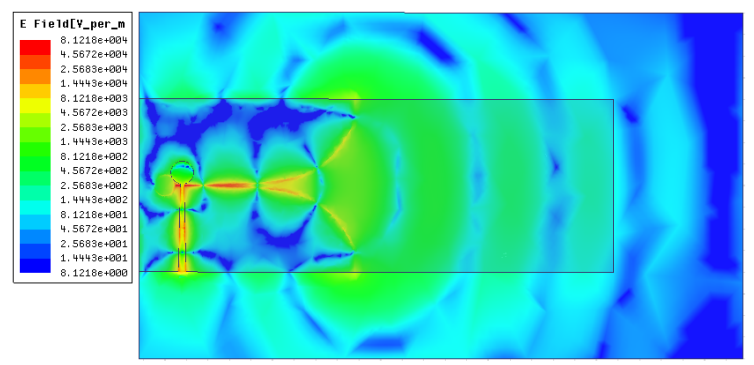

(a) 


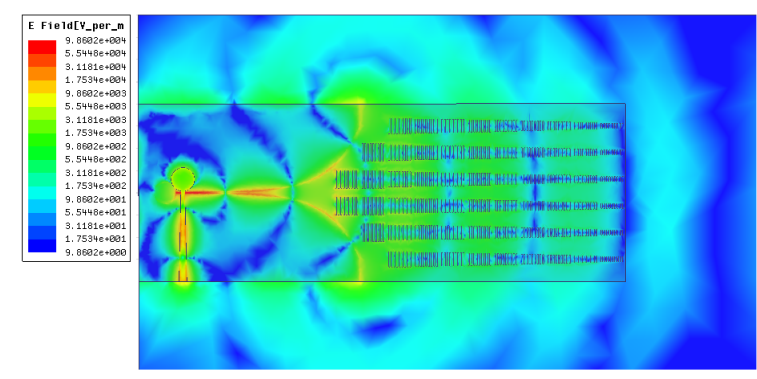

(b)

Figure 7: Electric field distributions at $9 \mathrm{GHz}$, (a) Reference TSA and (b) GRIN TSA.

Table 1 Variation of refractive index over $2-11 \mathrm{GHz}$

\begin{tabular}{cc}
\hline $\begin{array}{c}\text { Dimension, } m \\
(\mathrm{~mm})\end{array}$ & $\begin{array}{c}\text { Effective refractive } \\
\text { index, } \mathrm{n}\end{array}$ \\
\hline 4 & 1.53 to 1.67 \\
3.7 & 1.47 to 1.58 \\
3.4 & 1.42 to 1.5 \\
3.1 & 1.37 to 1.42 \\
2.9 & 1.34 to 1.38 \\
2.7 & 1.31 to 1.35 \\
2.4 & 1.29 to 1.32 \\
2.1 & 1.25 to 1.27 \\
1.8 & 1.23 to 1.24 \\
1.5 & 1.2 to 1.22 \\
1.1 & 1.18 to 1.19 \\
\hline
\end{tabular}

Fig. 6 shows the GRIN parallel-line metamaterial integrated tapered slot antenna. The proposed metamaterial is printed in front of the TSA with different refractive index values. The shape of the unit cell is designed to evenly match both TSA and air. The EM wave is propagating in an antenna with refractive index 1.88 (square root of the dielectric constant of the antenna substrate). Therefore, the metamaterial unit cell whose refractive index 1.53 is placed at the input slot area of the antenna and the lowest refractive index $(n=1.18)$ is integrated at the end of the antenna. In between the highest and lowest refractive indices $(n)$ of the metamaterial, the eight unit cell arrays are printed with descending order of $n$. The variation of refractive index on the length of $\mathrm{m}$ and frequency band is given in Table 1 .
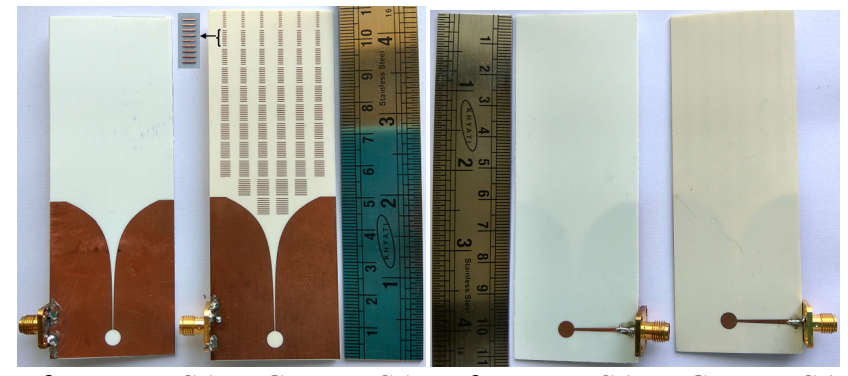

reference TSA

(a)

(b)

Figure 8: Photographs of the reference TSA and GRIN lens TSA (a) top view and (b) bottom view.
In order to understand the affect of the GRIN lens, the electric field distribution of the TSA with and without metamaterial is shown in Fig. 7. It is indicating that the GRIN helps to make the aperture field distribution much more uniform than the reference TSA. Because the phase velocity of the propagating wave in antenna is increased towards the output aperture width. So, the beam-width becomes narrower and the side lobe level is also reduced.

\section{Experimental Results and Discussions}

The top and bottom views of the fabricated reference TSA and GRIN loaded TSA are shown in Fig. 8. The comparison of the simulated and measured reflection coefficient $\left(\mathrm{S}_{11}\right)$ for reference and GRIN integrated TSA as illustrates in Fig. 9. Both antennas are giving below $-10 \mathrm{~dB}$ over frequency band 3 to $11 \mathrm{GHz}$, indicating that the impedance matching is good. But the $S_{11}$ is fluctuating near to $-10 \mathrm{~dB}$ over the frequency band 3 to $4.5 \mathrm{GHz}$. The GRIN parallel-line metamaterial does not affect broadband characteristics of the antenna. Similarly, the measured VSWR values are also plotted with respect to the frequency as shown in Fig. 10.

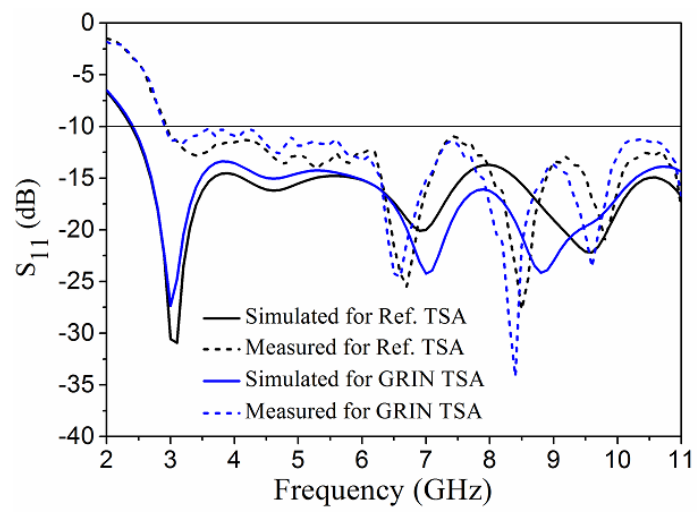

Figure 9: Reflection coefficient $\left(\mathrm{S}_{11}\right)$ of the reference (Ref.) and GRIN TSA

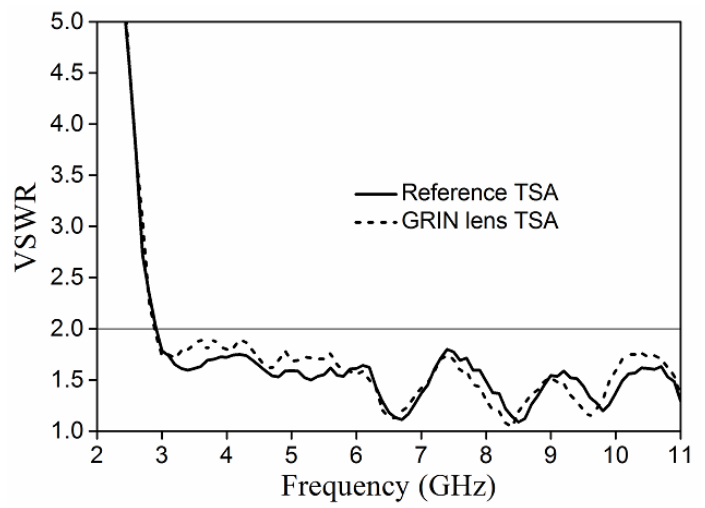

Figure 10: VSWR of the reference and GRIN TSA. 


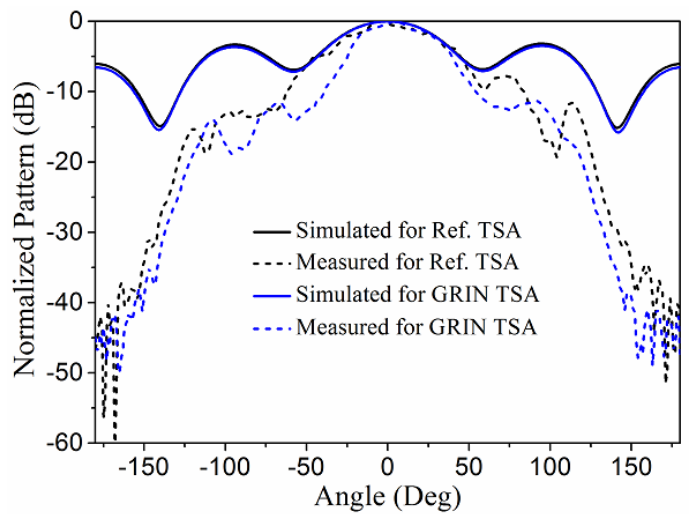

(a)

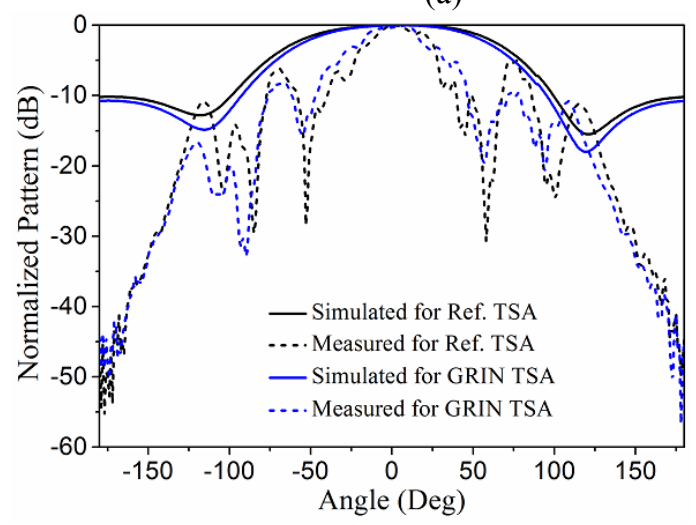

(b)

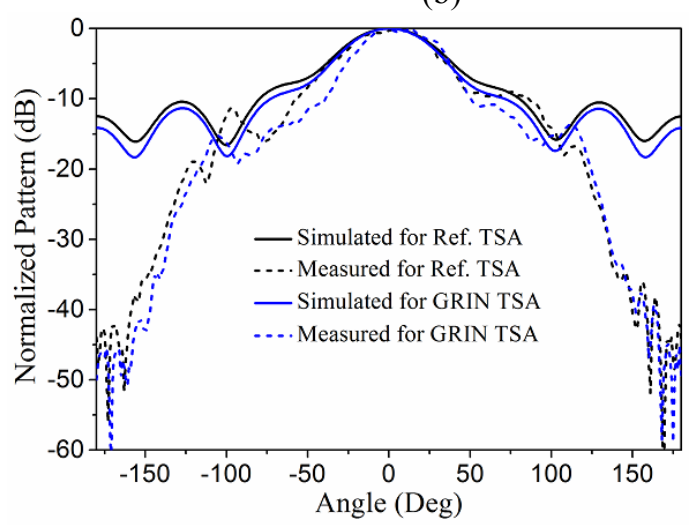

(c)

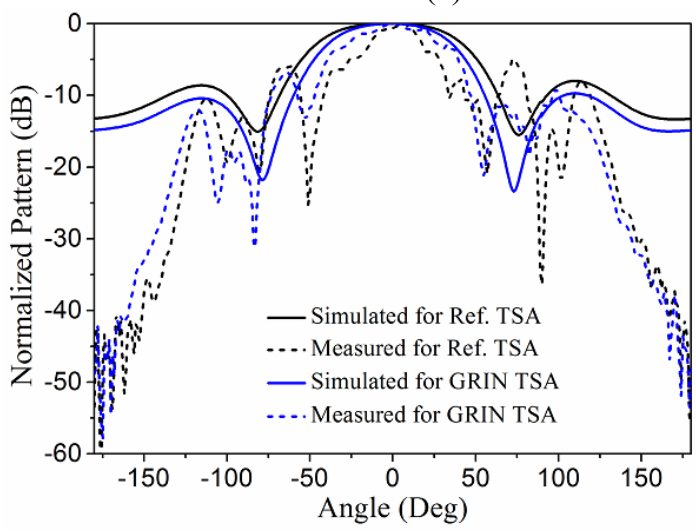

(d)

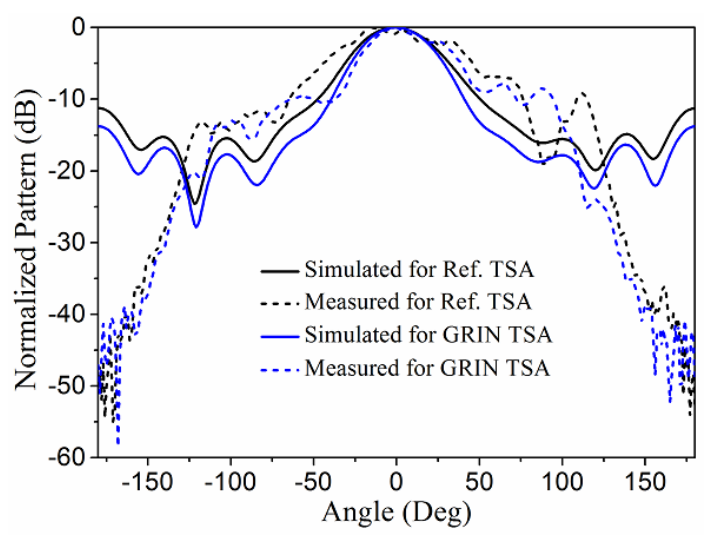

(e)

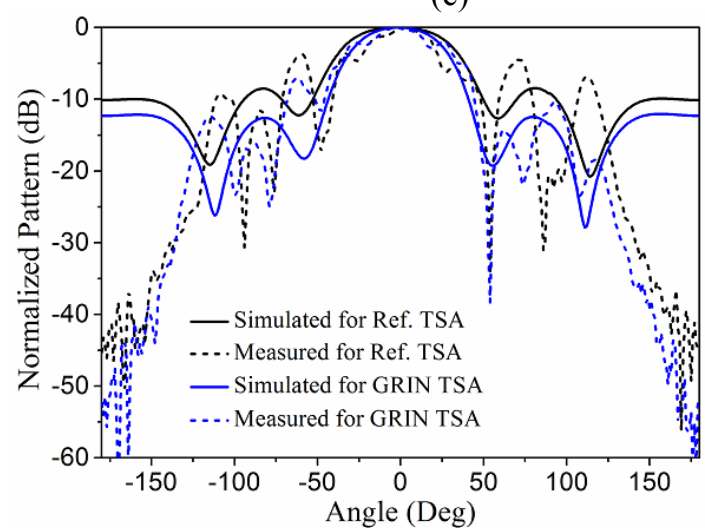

(f)

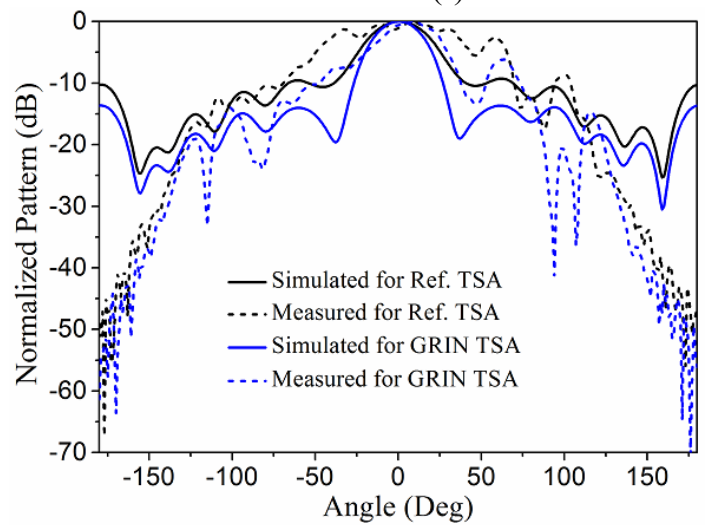

(g)

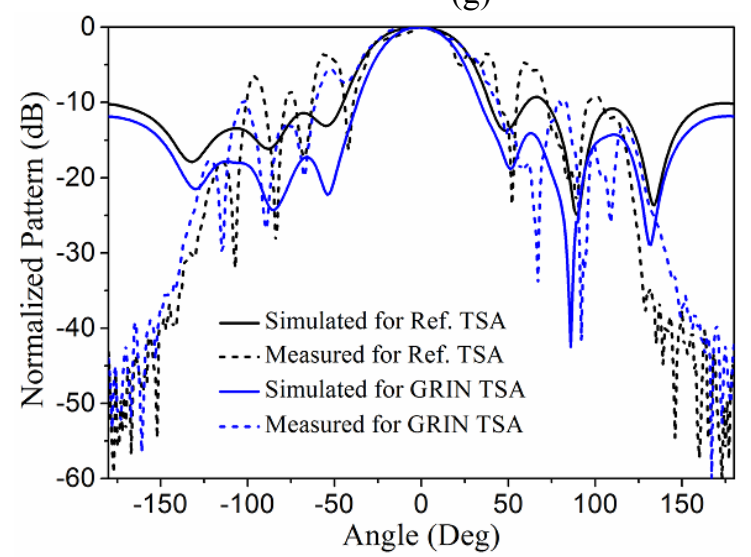

(h)

Figure 11: Measured radiation patterns of the reference TSA 
and GRIN TSA at (a) xy-plane for $3.5 \mathrm{GHz}$, (b) xz-plane for $3.5 \mathrm{GHz}$, (c) xy-plane for $6 \mathrm{GHz}$, (d) xz-plane for $6 \mathrm{GHz}$, (e) xy-plane for $8 \mathrm{GHz}$, (f) xz-plane for $8 \mathrm{GHz}$, (g) xy-plane for $10 \mathrm{GHz}$, (h) xz-plane for $10 \mathrm{GHz}$. The xy-plane and xzplane denotes $\mathrm{E}$ and $\mathrm{H}$-plane, respectively.

Fig. 11 shows the simulation and measured normalized radiation patterns of the fabricated two antennas with both E-plane (xy-plane) and H-plane (xz-plane) at $3.5 \mathrm{GHz}, 6$ $\mathrm{GHz}, 8 \mathrm{GHz}$ and $10 \mathrm{GHz}$. It is observed that for the GRIN TSA the half power beam width (HPBW) in the E-plane is narrower than reference TSA at high frequencies. So, it indicates that the radiation patterns becomes more directive. For the reference TSA, it is also seen that the fluctuation of side-lobes in the E and H-plane is high and reduced by using GRIN parallel-line metamaterial. Moreover, the simulated radiation patetrns are symmetric, but the measurement results are showing asymmetric pattern. The optimised simulation and measurement results are slightly different, because the 3D EM simulated designs are taken as ideal structure without any defect in antenna. But practically some minor defects might have crept in during PCB fabrication proces.

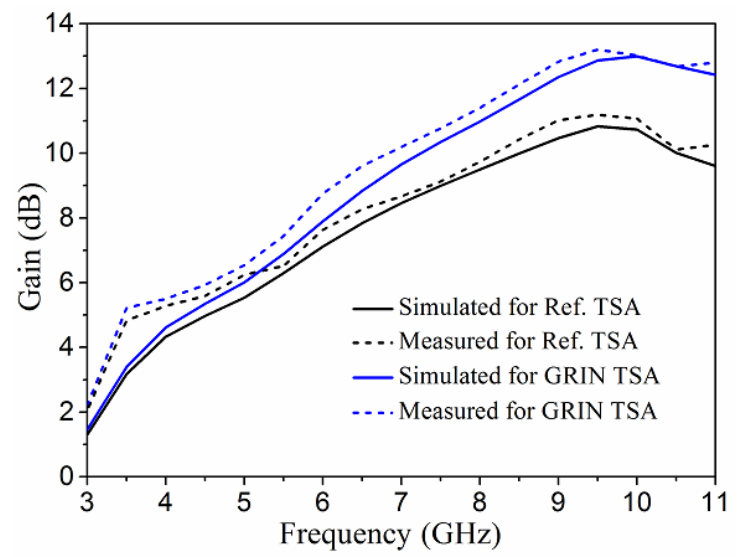

Figure 12: Measured gain of the reference TSA and GRIN TSA at the direction of $\mathrm{x}$ axis.

The simulated and measuread gain for the both antennas are compared which is depicted in Fig. 12. Compared to the simulation result, the measured gain is improved at low frequencies. The gain of the reference antenna is also significantly increased over frequency band $3-5 \mathrm{GHz}$ by using metamaterial. From the Fig. 12, it is clear that the gain of the reference TSA is actively increased at high frequencies by using GRIN metamaterial and varies from 11.1-13.2 dB at $9.5 \mathrm{GHz}$.

Due to the GRIN metamaterial the proposed UWB antenna size is increased. Therefore, the time domain characteristics are also determined for the antenna. The time domain characteristics of the reference and GRIN TSA is shown in Fig. 13. To measure the group delay, two identical antennas are placed opposite direction with the distance of $30 \mathrm{~cm}$ and connected with the ports of vector network analyzer. The fluctuation of group delay for reference TSA is more stable than GRIN TSA. The comparison between proposed antenna and published work in terms of size, peak gain increment is listed in Table 2. The proposed antenna has less size compared with references [2], [8] and gain is increased compared with [9].

Table 2 Comparison between proposed antenna and existing work

\begin{tabular}{ccc}
\hline References & $\begin{array}{c}\text { Size } \\
\left(\mathrm{mm}^{2}\right)\end{array}$ & Peak gain $(\mathrm{dB})$ \\
\hline$[2]$ & $140 \times 80$ & 14 \\
{$[3]$} & $77 \times 42$ & 12 \\
{$[8]$} & $190 \times 130$ & $15 \mathrm{dBi}$ \\
{$[9]$} & $36 \times 36$ & $8 \mathrm{dBi}$ \\
Proposed antenna & $110 \times 40$ & 13 \\
\hline
\end{tabular}

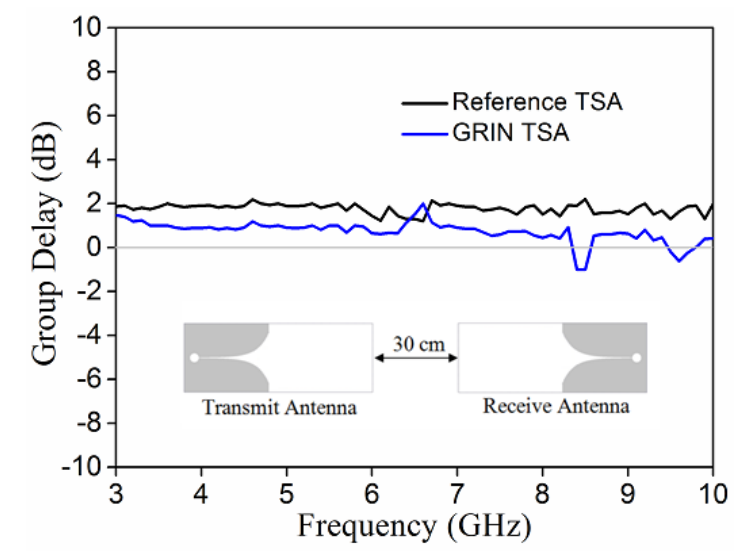

Figure 13: Measured group delay performance of the GRIN TSA.

\section{Conclusions}

This paper introduces a broadband gradient index metamaterial which is designed by using parallel-line structures. The proposed material is integrated with reference TSA to enhance the gain of the antenna. As a result, the E-plane and H-plane have narrow and broad HPBWs, respectively. The measured results of the fabricated antenna are showing that the gain of the reference antenna is increased by using metamaterial. The side lobe level of the reference antenna is also reduced. Therefore, the proposed metamaterial is useful as end-fire antenna for ultra-wideband communication systems.

\section{References}

[1] Gibson P. J., The Vivaldi Aerial, 9th European Microwave Conference, 101-105, 1979.

[2] B. Zhou and T. J. Cui, Directivity enhancement to Vivaldi antennas using compactly anisotropic zeroindex metamaterials, IEEE Antennas Wirel. Propag. Lett., 10: 326-329, 2011. 
[3] L. Chen, Z. Lei, R. Yang, J. Fan, and X. Shi, A broadband artificial material for gain enhancement of antipodal tapered slot antenna, IEEE Trans. Antennas Propag., 63: No. 1, 395-400, 2015.

[4] L. M. Zhong and T. J. Cui, Experimental realization of a broadband bend structure using gradient index metamaterials, Opt. Exp., 17: No. 20,18354-18363, 2009.

[5] X. Chen, T. M. Grzegorczyk, B. I. Wu, J. Pacheco, and J. A. Kong, Robust method to retrieve the constitutive effective parameters of metamaterials, Phys. Rev. E, 70: Feb. 2004, Art. ID 016608.

[6] E. Gazit, Improved design of the Vivaldi antenna, Inst. Elect. Eng. Proc., 135: No. 2, 89-92, 1988.

[7] Schüppert B., Microstrip/slot line transition: modeling and experimental investigation, IEEE Trans. Microw. Theory Tech., 36: No. 8, 1272-1282, 1988.

[8] A. Dhouibi, S. N. Burokur, A. de Lustrac and A. Priou, Low-profile substrate-integrated lens antenna using metamaterials, IEEE Antennas Wireless Propag. Lett., 12: 43-46, 2013.

[9] K. Ma, Z. Q. Zhao, J. N. Wu, M. S. Ellis and Z. P. Nie, A printed Vivaldi Antenna with improved radiation patterns by using two pairs of Eye-Shaped Slots for UWB applications, Progress In Electromagnetics Research, 148: 63-71, 2014.

[10] A. De Oliveira, M. Perotoni, S. Kofuji, and J. Justo, A palm tree antipodal Vivaldi antenna with exponential slot edge for improved radiation pattern, IEEE Antennas Wireless Propag. Lett., 14: 1334-1337, 2015.

[11] de Oliveira, Alexandre M., João F. Justo, Marcelo B. Perotoni, Sérgio T. Kofuji, Alfredo G. Neto, Regis C. Bueno, and Henri Baudrand, A high directive Koch fractal Vivaldi antenna design for medical near-field microwave imaging applications, Microwave Opt. Technol. Lett., 59, No. 2: 337-346, 2017.

[12] Singha Rahul and Vakula Damera, Directive beam of the monopole antenna using broadband gradient refractive index metamaterial for ultra-wideband application, IEEE Access, 5: 9757-9763, 2017. 